\title{
Index assignment for MDVQ over memoryless binary symmetric channel with packet erasure
}

\author{
Jeng-Shyang Pan $^{1}$, Yao Zhao ${ }^{2}$, Kang K. Yen ${ }^{3}$, and Yi-Chih Hsin ${ }^{1}$ \\ ${ }^{1}$ Dept. Electronic Eng., National Kaohsiung University of Applied Sciences, Kaoh- \\ siung, Taiwan, R. O. C. \\ ${ }^{2}$ Institute of Information Science, Beijing Jiaotong University, P. R. China \\ ${ }^{3}$ Dept. of Electrical and Computer Engineering, Florida International University, \\ U.S.A.
}

Abstract: Genetic algorithm is applied to index assignment for multiple description vector quantization (MDVQ) over memoryless binary symmetric channel (BSC) with packet erasure. The cost evaluation function is presented based on this hybrid model. Simulation results based on the bit error rate for binary symmetric channel and the packet erasure rate demonstrate the usefulness of the proposed optimization scheme.

Keywords: vector quantization, MDVQ, binary symmetric channel Classification: Science and engineering for electronics

\section{References}

[1] V. Vaishampayan, "Design of multiple description scalar quantizers," IEEE Trans. Inform. Theory, vol. 39, no. 3, pp. 821-834, 1993.

[2] A. Gavrilesc, A. Munteanu, P. Schelkens, and J. Cornelis, "Embedded multiple description scalar quantisers," Electron. Lett., vol. 39, no. 13, pp. 979-980, 2003.

[3] V. Vaishampayan, N. Sloane, and S. Servetto, "Multiple-description vector quantization with lattice codebooks: design and analysis," IEEE Trans. Inform. Theory, vol. 47, no. 5, pp. 1718-1734, 2001.

[4] V. Goyal, J. Kelner, and J. Kovacevic, "Multiple description vector quantization with a coarse lattice," IEEE Trans. Inform. Theory, vol. 48, no. 3, pp. 781-788, 2003.

[5] N. Gortz and P. Leelapornchai, "Optimization of the index assignments for multiple description vector quantizers," IEEE Trans. Commun., vol. 51, no. 3, pp. 336-340, 2003.

[6] D. Golberg, "Genetic algorithm in search, optimization and machine learning," Addison-Wesley Publishing Company, 1989.

[7] Y. Linde, A. Buzo, and R. Gray, "An algorithm for vector quantizer design," IEEE Trans. Commun., vol. 28, no. 1, pp. 84-95, 1980. 


\section{Introduction}

Multiple descriptions coding (MDC) $[1,2]$ has been shown to be a robust scheme for communication based on the diversity to overcome channel impairments. The basic idea is to separate and code the information into several parts transmitted through several channels. Each part of the transmitted information is called description. For packet loss free, the transmitted information can be fully recovered through the received descriptions in the decoder. Due to the channel impairments, some descriptions might be loss, the decoded information with a limited distortion level can be guaranteed even only part of the descriptions are received. The application of two descriptions for vector quantizer has been investigated $[3,4]$ and the index assignment has been shown to be useful to reduce the distortion for multiple description vector quantization (MDVQ) based on the packet erasure for unreliable channels [5]. However, the evaluation function in [5] is only based on packet erasure. Bit error may be happen due to noisy channels. In this letter, both the packet erasure and the bit error for memoryless binary symmetric channel are considered together to generate the evaluation function for this hybrid model. The optimization of index assignment based on the genetic algorithm [6] can efficiently overcome the channel impairments of this hybrid model.

\section{Binary symmetric channel with packet erasure}

Briefly, we only draw MDVQ with two channels shown in Fig. 1. Given the packet erasure rate for the $j$ th channel, $p_{P E R_{j}}$, the packet erasure channel model for receiving the index $\bar{i}_{j}$ after sending the index $i_{j}$ can be expressed as

$$
P\left(\bar{I}_{j}=\bar{i}_{j} \mid I_{j}=i_{j}\right)=\left\{\begin{array}{ll}
p_{P E R_{j}}, & \text { if } \bar{i}_{j}=\phi \\
1-p_{P E R_{j}}, & \text { if } \bar{i}_{j}=i_{j} \\
0, & \text { else }
\end{array},\right.
$$

where $\phi$ means packet erasure.

Assume $H\left(\bar{i}_{j}, i_{j}\right)$ is the Hamming distance between the sending index $i_{j}$ and the receiving index $\bar{i}_{j}$ and the bit string length of $i_{j}$ and $\bar{i}_{j}$ is $L$. Given

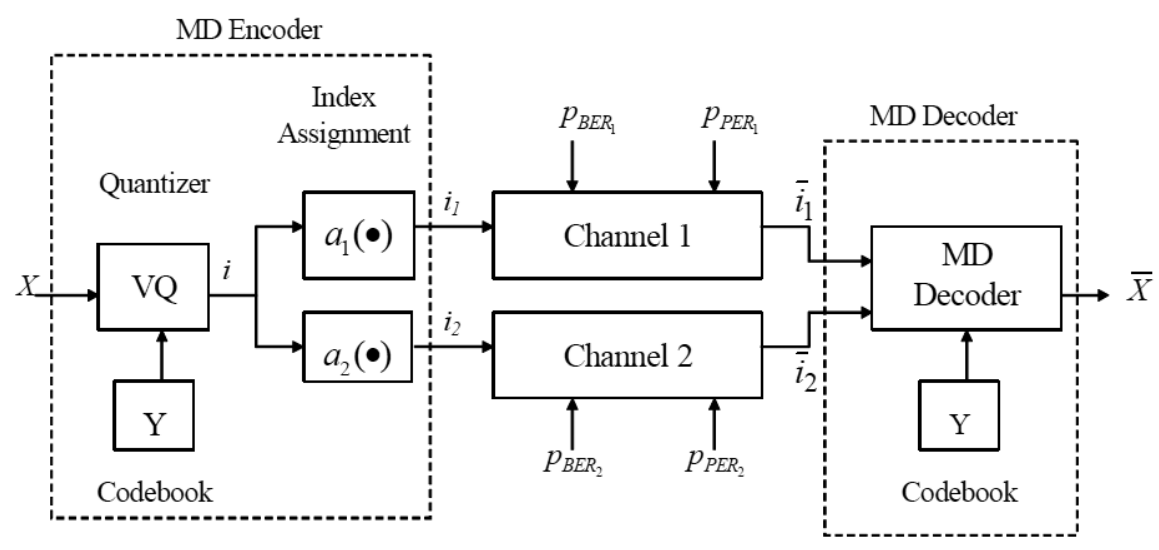

Fig. 1. MDVQ with two channels 
the bit error rate for the $j$ th channel, $p_{B E R_{j}}$, the binary symmetric channel model can be expressed as

$$
P\left(\bar{I}_{j}=\bar{i}_{j} \mid I_{j}=i_{j}\right)=p_{B E R_{j}}{ }^{H\left(\bar{i}_{j}, i_{j}\right)}\left(1-p_{B E R_{j}}\right)^{\left(L-H\left(\bar{i}_{j}, i_{j}\right)\right)}
$$

Combine Eq. (1) and Eq. (2), the mathematical model for binary symmetric channel with packet erasure can be formulated as

$P\left(\bar{I}_{j}=\bar{i}_{j} \mid I_{j}=i_{j}\right)= \begin{cases}p_{P E R_{j}}, & \text { if } \bar{i}_{j}=\phi \\ p_{B E R_{j}}{ }^{H\left(\bar{i}_{j}, i_{j}\right)}\left(1-p_{B E R_{j}}\right)^{\left(L-H\left(\bar{i}_{j}, i_{j}\right)\right)}, & \text { else }\end{cases}$

Assume $N$ and $K$ are the number of codevectors and the number of descriptions, respectively. Denote $S_{j}$ the partitioned set for the $j$ th channel and $a_{j}(\bullet)$ the mapping function for the index assignment in the $j$ th channel. $y_{\lambda}$ is the codevector, $\lambda=0, \ldots, N-1$, and $\bar{X}$ is the reconstructed vector after decoding the descriptions. $d(\bullet, \bullet)$ is the distortion between two input vectors. The design of the MDVQ is to minimize the channel distortion as following:

$$
\begin{aligned}
D= & \sum_{\lambda=0}^{N-1} P(I=\lambda) \sum_{\forall \overline{v i}_{1} \in S_{1}} \cdots \sum_{\forall \bar{i}_{K} \in S_{K}} d\left(y_{\lambda}, \bar{X}\left(\bar{i}_{1}, \cdots, \bar{i}_{K}\right)\right) \\
& \cdot \prod_{j=1}^{K} P\left(\bar{I}_{j}=\bar{i}_{j} \mid I_{j}=a_{j}(\lambda)\right)
\end{aligned}
$$

If the codebook size is 64 and the number of indices in each channel is 16 , the design of index assignment is to locate 64 codevecors in the 256 entries (i.e. $16 \times 16$ ). Obviously it is a combinatorial optimization problem. The genetic algorithm can be applied to assign the indices for all channels so as to minimize the channel distortion in Eq. (4).

\section{Simulation results}

1000 training data and 1000 test data with two dimensions are generated from the Gaussian source with zero mean and unit variance, respectively. The Generalized Lloyd algorithm [7] is used to generate 64 codevectors from the training data. Experiments are based on two channels and the size of indices for each channel is 16. The first experimental results shown in Fig. 2, is assumed the packet erasure rate is 0 and simulate the SNR for the bit error rate from 0 to 0.5 for the proposed method and the diagonal method [1]. The second experiment is based on the assumption that the bit error rate is 0 and the packet erasure rate is from 0 to 1.0. Simulation results are shown in Fig. 3. All the experiments confirm the proposed method is useful for this hybrid model.

\section{Conclusion}

A hybrid model based on the memoryless binary symmetric channel and packet erasure channel is studied. The evaluation function for this model 


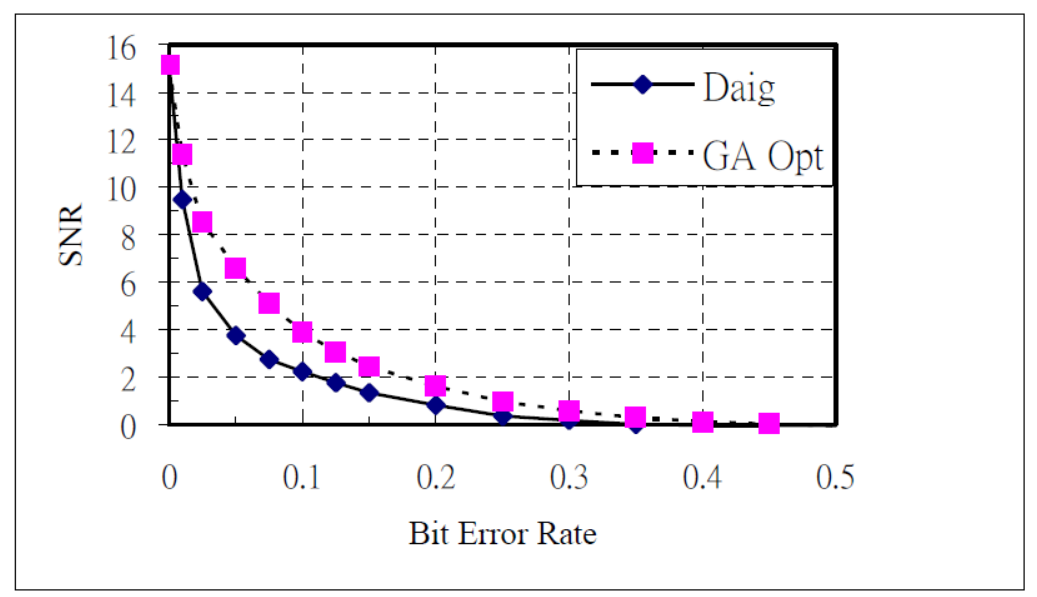

Fig. 2. Index Assignment Results for $\mathrm{PER}=0, \mathrm{BER}=$ $0.0 \sim 0.5$

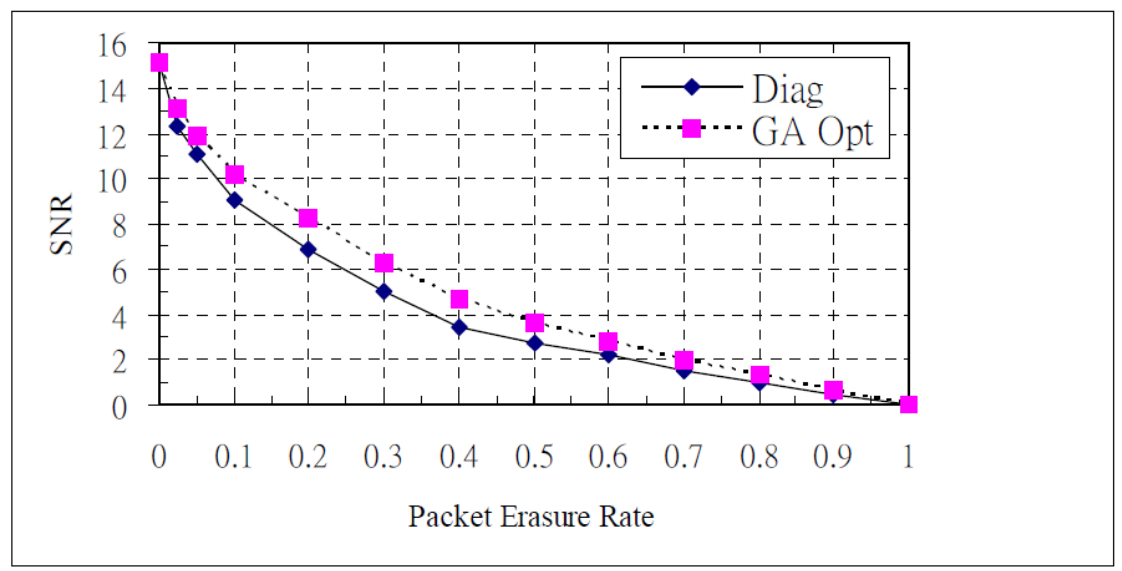

Fig. 3. Index Assignment Results for $\mathrm{BER}=0, \mathrm{PER}=$ $0.0 \sim 1.0$

is presented. The presented hybrid model and optimization scheme can not only improve the signal to noise ratio for the packet erasure channel but also reduce the channel distortion due to the bit errors in the noisy channels. Simulation results based on genetic algorithm demonstrate the robustness of the proposed method.

\section{Acknowledgments}

This work is partially funded by Dept. of Defense (USA) under award number H98230-04-C-0460, Dept. of Transportation (USA) under project number FL29-7102-00 and National Science Council (Taiwan, ROC) under grant number NSC 93-2213-E-151-016. 